\title{
ALMOST PERIODIC SOLUTIONS FOR SYSTEMS OF DIFFERENTIAL EQUATIONS NEAR POINTS OF NONLINEAR FIRST APPROXIMATION
}

\section{GEORGE SEIFERT ${ }^{1}$}

1. Introduction. Sufficient conditions for the existence of almost periodic (a.p. for short) solutions of systems of the form

$$
\dot{x}_{i}=\sum_{j=1}^{n} a_{i j} x_{j}+r\left(x_{1}, \cdots, x_{n}, t\right), \quad i=1,2, \cdots, n,
$$

are known provided the characteristic values of the matrix $a=\left(a_{i j}\right)$ have nonzero real parts; cf., for example, [1]. Here the $r_{i}$ are uniformly a.p. in $t$ for $\left(x_{i}, \cdots, x_{n}\right)$ in some region of Euclidean $n$-space. Amerio [3] has obtained such conditions for somewhat more specialized systems where some characteristic values of $A$ may be pure imaginary; however, all must be distinct from zero.

In case $n=2$, sufficient conditions for the existence, uniqueness, and asymptotic stability of a.p. solutions are known even if one of the characteristic values of $A$ is zero. In fact, the system considered in $[2]$ is

$$
\begin{aligned}
& \dot{x}=y-F(x), \\
& \dot{y}=-g(x)+p(t),
\end{aligned}
$$

where $F(0)=g(0)=0$, and the derivatives $F^{\prime}(x)$ and $g^{\prime}(x)$ are such that $F^{\prime}(x)>0$, and $g^{\prime}(x) \geqq 0$ where $g^{\prime}(x)=0$ only when $x=0$. In this paper, a system of essentially this type is considered; we require now that $g^{\prime}(x) \leqq 0$ where $g^{\prime}(x)=0$ only when $x=0$, and if $g^{\prime}(0)=0$, then $F^{\prime}(0)=0$. We prove an existence theorem for a unique a.p. solution which in case $F^{\prime}(x)>0$ for $x \neq 0$, has certain stability properties. We observe that this system is equivalent to the single second order equation:

$$
\ddot{x}+F^{\prime}(x) \dot{x}+g(x)=p(t) .
$$

Throughout this paper, conventional topological definitions and notation is used; i.e., a region is an open connected plane set; the closure, union, intersection of sets are denoted respectively by $\bar{R}$, $R \cup S, R \cap S$ where $R$ and $S$ are sets; $p \in S$ means $p$ is a member of $S$, etc.

Received by the editors July 15, 1959.

${ }^{1}$ Sponsored by the Office of Ordnance Research, U. S. Army. 
2. The existence theorem. We consider the system

$$
\begin{aligned}
& \dot{x}=y-F(x), \\
& \dot{y}=g(x)+p(t) ;
\end{aligned}
$$

here (i) $p(t)$ is a.p. and $k=\max |p(t)|$;

(ii) $g(0)=F(0)=0$;

(iii) there exist numbers $a, b, a<0<b$, such that $g(x)>k$ for $x \geqq b$, $g(x)<-k$ for $x \leqq a$;

(iv) $g^{\prime}(x)$ and $F^{\prime}(x)$ are continuous, and $g^{\prime}(x) \geqq 0$ for $a \leqq x \leqq b$. We define

$$
\begin{aligned}
& \phi(x, \xi)= \begin{cases}(F(x+\xi)-F(x)) / \xi, & \xi \neq 0, \\
F^{\prime}(x), & \xi=0 ;\end{cases} \\
& h(x, \xi)= \begin{cases}(g(x+\xi)-g(x)) / \xi, & \xi \neq 0, \\
g^{\prime}(x), & \xi=0 ;\end{cases}
\end{aligned}
$$

and

$$
D(x, \xi)=\phi^{2}(x, \xi)-4 h(x, \xi) .
$$

In terms of these definitions we state and prove the following

Theorem. If $D(x, \xi) \leqq 0$ for $a \leqq x \leqq b, a \leqq x+\xi \leqq b$, and $D(x, \xi)=0$ only if $\xi=0$, then system (2) has a unique a.p. solution. If also $\phi(x, \xi)>0$ for $\xi \neq 0$, then this a.p. solution is asymptotically stable as $t \rightarrow+\infty$ with respect to a class $\Sigma_{1}$ of solutions of (2), and asymptotically stable as $t \rightarrow-\infty$ with respect to a class $\Sigma_{2}$ of solutions of (2).

The proof of this theorem is based on a series of lemmas which are stated and proved after the following remarks.

REMARK 1. $g^{\prime}(0)=F^{\prime}(0)=0$ is possible; hence, if $(2)$ is considered as a special case of (1), the matrix $A$ is in that case given by

$$
A=\left(\begin{array}{ll}
0 & 1 \\
0 & 0
\end{array}\right)
$$

the characteristic values of which are both zero. Hence, the system is markedly nonlinear at the origin.

REMARK 2. If $a \leqq x_{n} \leqq b, a \leqq x_{n}+\xi_{n} \leqq b$ for $n=1,2, \cdots$, then $D\left(x_{n}, \xi_{n}\right) \rightarrow 0$ as $n \rightarrow \infty$ implies $\xi_{n} \rightarrow 0$ as $n \rightarrow \infty$; this clearly follows from the hypotheses of the theorem, and continuity.

The following lemmas concern themselves with the system

$$
\begin{aligned}
& \dot{x}=y-F(x), \\
& \dot{y}=g(x)+e(t),
\end{aligned}
$$


where $e(t)$ is in the closure of the set of functions $\{p(t+h)\},|h|<\infty$, the closure being with respect to the uniform norm on $|t|<\infty$. Clearly $|e(t)| \leqq k$ for all $t$. Conditions on $g(x)$ and $F(x)$ as before (involving $k$ ) are presumed.

We consider the region $R$ of the $(x, y)$ plane bounded by the lines $y=m x \pm c, x=a, x=b$; here $m>0$ is fixed but arbitrary, and $c>0$ is such that for $a \leqq x \leqq b$ and $n=1,2$ :

$$
m>\frac{g(x)+(-1)^{n} k}{(-1)^{n} c+m x-F(x)}
$$

and

$$
\left|m x+(-1)^{n} c\right|>F(x) .
$$

We denote by $\Gamma$ the boundary of $R$, and by $\Gamma_{a}$ and $\Gamma_{b}$ the subsets of $\Gamma$ respectively defined by $x=a, m a-c<y<F(a)$ and $x=b, F(b)<y$ $<m b+c$.

Lemma 1. Let $(x(t), y(t))$ be a solution of (3) such that $\left(x\left(t_{0}\right), y\left(t_{0}\right)\right) \in R$ and $\left(x\left(t_{2}\right), y\left(t_{2}\right)\right) \notin \bar{R}$ for some $t_{2}>t_{0}$. Then there exists a $t_{1}, t_{0}<t_{1}<t_{2}$, such that $\left(x\left(t_{1}\right), y\left(t_{1}\right)\right) \in \Gamma_{a} \cup \Gamma_{b}$, and such that $(x(t), y(t)) \notin R$ for $t>t_{1}$. Also, either $x\left(t_{2}\right)<a$ or $x\left(t_{2}\right)>b$, in the former case, $\left(x\left(t_{1}\right), y\left(t_{1}\right)\right) \in \Gamma_{a}$, in the latter case $\left(x\left(t_{1}\right), y\left(t_{1}\right)\right) \in \Gamma_{b}$.

Proof. From (vi) above, the graph of $y=F(x)$ for $a<x<b$ is in $R$. It follows from (v) that the slope of $\Gamma$ along the lines $y=m x \pm c$ is greater than the slope of the trajectory of any solution of (3) at points on these parts of $\Gamma$, i.e., trajectories of (3) cannot leave $R$ there as $t$ increases.

Since $y>F(x)$ implies $\dot{x}>0$ and $y<F(x)$ implies $\dot{x}<0$, it follows that trajectories of (3) above $y=F(x)$ move to the right as $t$ increases, while those below $y=F(x)$ move to the left. Hence, trajectories cannot leave $R$ on the part of $\Gamma$ on $x=a$ above $y=F(x)$ and on $x=b$ below $y=F(x)$.

It remains only to consider the trajectories at points $(a, F(a))$ and $(b, F(b))$ of $\Gamma$. In fact, if the trajectory of a solution $(x(t), y(t))$ of $(3)$ is such that $x\left(t_{0}\right)=b_{1} \geqq b, y\left(t_{0}\right)=F\left(b_{1}\right)$, then $\dot{x}\left(t_{0}\right)=0$ and $\dot{y}\left(t_{0}\right)>0$. Hence this trajectory has a vertical tangent at $\left(b_{1}, F\left(b_{1}\right)\right)$, is on the right side of this tangent for $t \neq t_{0}$, and moves up across the graph of $y=F(x)$ there as $t$ increases. It follows that any trajectory leaving $R$ on $\Gamma_{b}$ cannot return to $R$ for $t$ sufficiently large.

A similar discussion applies to the situation at points of the form $\left(a_{1} F\left(a_{1}\right)\right)$ where $a_{1} \leqq a$, except that at such points, trajectories of (3) 
cross down over $y=F(x)$ as $t$ increases; we omit the details, and the proof of the lemma is complete.

Lemma 2. There exists a solution $(x(t), y(t))$ of (3) such that $(x(t), y(t)) \in \bar{R}$ for all $t \geqq 0$.

Proof. Let $p$ be a point of $(x, y)$ plane; then we will denote by $\mu(p, t)$ the point $(x(t), y(t))$ where $(x(t), y(t))$ is the solution of $(3)$ such that $(x(0), y(0))=p$. We also denote by $p_{1}$ and $p_{2}$ the points of $\Gamma$ given by $(a, F(a))$ and $(b, m b+c)$ respectively and by $\Gamma_{1}$ the part of $\Gamma$ between these points but above the curve $y=F(x)$. Let the set of points on $\Gamma_{1}$ be ordered so that if $p$ and $q$ are distinct points on $\Gamma_{1}$, then $p>q$ means that the distance along $\Gamma_{1}$ from $p$ to $p_{2}$ is greater than the distance along $\Gamma_{1}$ from $q$ to $p_{2}$.

Consider the set $S_{b}$ of points on $\Gamma_{1}$ such that if $p \in S_{b}$, then for each $q \in \Gamma_{1}, q<p$, there exists a $T=T(q) \geqq 0$ such that $\mu(q, T) \in \Gamma_{b}$. This set is clearly nonempty and in fact contains an open interval of $\Gamma_{1}$ which has $p_{2}$ as its end point. Similarly, $\Gamma_{1}-S_{b}$ contains an interval having $p_{1}$ as end point. Since $S_{b}$ is bounded above, it has a least upper bound (l.u.b. for short), say $q_{1} \neq p_{1}$. Clearly $q_{1} \neq p_{2}$. We shall show that $\mu\left(q_{1}, t\right) \in R$ for all $t>0$. For if not, then by Lemma 1 there exists a $T_{1} \geqq 0$ such that $\mu\left(q_{1}, T_{1}\right) \in \Gamma_{a} \cup \Gamma_{b}$. Suppose $\mu\left(q_{1}, T_{1}\right) \in \Gamma_{a}$, and let $t_{1}>0$ be fixed but arbitrary. Then by Lemma 1 there exists a region $T_{a}$ in the half plane $x<a$ such that $\mu\left(q_{1}, T_{1}+t_{1}\right) \in R_{a}$. By continuity of solutions of (3) as functions of their initial conditions, there exists a region $S_{1}$ such that $q_{1} \in S_{1}$, and if $p \in S_{1} \cap \Gamma_{1}$, there $\mu\left(p, T_{1}+t_{1}\right) \in R_{a}$, and hence, by Lemma 1 there exists a $\theta, 0<\theta<1$, such that $\mu\left(p, \theta\left(T_{1}+t_{1}\right)\right) \in \Gamma_{a}$. Since $S_{1} \cap \Gamma_{1}$ is an open interval of $\Gamma_{1}$ containing $q_{1}$, this contradicts the assumption that $q_{1}$ is a l.u.b. for $S_{b}$.

The case $\mu\left(q_{1}, T_{2}\right) \in \Gamma_{b}$ for some $T_{2} \geqq 0$ is ruled out in the same way; we omit the details.

Lemma 3. There exists a solution $(x(t), y(t))$ of (3) such that $(x(t), y(t)) \in R$ for all $t$.

Proof. From Lemma 2, there exists a solution $\left(x_{0}(t), y_{0}(t)\right)$ of $(3)$ such that $(x(t), y(t)) \in \bar{R}$ for $t \geqq 0$. From a result of Amerio [3], there exists a solution $(x(t), y(t))$ of (3) such that $(x(t), y(t)) \in R$ for all $t$. This proves the lemma.

Lemma 4. If $(x(t), y(t)) \in R$ for all $t$, then this solution is unique.

Proof. Suppose there exists a solution $\left(x_{1}(t), y_{1}(t)\right)$ of (3) such that $\left(x_{1}(t), y_{1}(t)\right) \in R$ for all $t$, and such that if $\xi(t)=x_{1}(t)-x(t)$ and $\eta(t)$ 
$=y_{1}(t)-y(t)$, then $(\xi(t), \eta(t)) \neq(0,0)$. From (3) it follows that $(\xi(t), \eta(t))$ is a solution of

$$
\begin{aligned}
& \xi=\eta-\phi(x(t), \xi) \xi, \\
& \dot{\eta}=h(x(t), \xi) \xi .
\end{aligned}
$$

It follows that $(\xi \eta)=Q(\xi, \eta)$ where $Q(\xi, \eta)=\eta^{2}+h \xi^{2}-\phi \xi \eta$, and $h$ and $\phi$ are as defined just previous to the statement of the theorem.

Since $D(x(t), \xi(t)) \leqq 0$ for all $t$, it follows that $(\xi \eta) \geqq 0$; i.e., $\xi \eta$ is nondecreasing in $t$. But since $\xi$ and $\eta$ are, by hypothesis, bounded, it follows that there exist numbers $c$ and $d$ such that $c \leqq d$, that $\xi \eta \rightarrow c$ as $t \rightarrow-\infty$, and that $\xi \eta \rightarrow d$ as $t \rightarrow+\infty$.

Suppose first $c=d$; then clearly $\xi \eta=c=d$; hence $(\xi \eta)=Q(\xi, \eta)=0$ for all $t$. But $Q(\xi, \eta)=0$ clearly implies $D(x, \xi)=0$, and from the hypotheses of the theorem, $\xi=0$. But then $Q(0, \eta)=0$ implies $\eta=0$. Hence $c=d$ implies $(\xi, \eta)=(0,0)$, a contradiction.

Suppose $c<d, d \neq 0$. Since $\xi \eta \rightarrow d$ as $t \rightarrow+\infty$, there exists a sequence $\left\{t_{n}\right\}, n=1,2, \cdots, t_{n} \rightarrow+\infty$, such that for $t=t_{n} \rightarrow+\infty,(\xi \eta) \rightarrow 0$; i.e., $Q\left(\xi\left(t_{n}\right), \eta\left(t_{n}\right)\right) \rightarrow 0$ as $n \rightarrow \infty$. Since $\eta(t)$ is bounded for all $t, \xi \eta \rightarrow d \neq 0$ implies that $\xi\left(t_{n}\right)$ cannot approach zero as $n \rightarrow \infty$. Hence, there exists a subsequence $\left\{t_{n_{i}}\right\}$ of $\left\{t_{n}\right\}$ such that $t_{n_{i}} \rightarrow+\infty$ as $i \rightarrow \infty$, and that $\left|\xi\left(t_{n_{i}}\right)\right| \geqq \rho>0$ for some constant $\rho$. But $Q\left(\xi_{i}, \eta_{i}\right) \rightarrow 0$ implies $D\left(x_{i}, \xi_{i}\right)$ $\rightarrow 0$ is $i \rightarrow \infty$, here $x_{i}=x\left(t_{n_{i}}\right)$, and $\xi_{i}$ and $\eta_{i}$ are similarly defined. Hence by Remark $2, \xi_{i} \rightarrow 0$ as $i \rightarrow \infty$, a contradiction.

Finally suppose $d=0$ and $c<0$. Then there exists a sequence $\left\{t_{n}\right\}$, $n=1,2, \cdots, t_{n} \rightarrow-\infty$, such that $Q\left(\xi\left(t_{n}\right), \eta\left(t_{n}\right)\right) \rightarrow 0$ and $n \rightarrow \infty$. Again the fact that $\xi(t)$ cannot approach zero on any subsequence of $\left\{t_{n}\right\}$ leads to a contradiction as before; we omit the details.

Hence, the solution $(x(t), y(t)) \in R$ for all $t$ is unique, and the lemma is proved.

Lemma 5. If $\Gamma_{a}$ and $\Gamma_{1}$ are as defined in Lemmas 1 and 2, and if $(x(t), y(t))$ is the unique solution of (3) such that $(x(t), y(t)) \in R$ for all $t$, then there exist nonempty closed sets $\Gamma_{+} \subset \Gamma_{1}$ and $\Gamma_{-} \subset \Gamma_{a}$ such that if $\left(x_{1}(t), y_{1}(t)\right)$ is a solution of $(3)$ and $(\xi(t), \eta(t))$ are as defined in Lemma 4 , then

(a) $\quad\left(x_{1}(0), y_{1}(0)\right) \in \Gamma_{+}$implies $(\xi(t), \eta(t)) \rightarrow(0,0)$ as $t \rightarrow+\infty$;

(b) $\quad\left(x_{1}(0), y_{1}(0)\right) \in \Gamma_{-}$implies $(\xi(t), \eta(t)) \rightarrow(0,0)$ as $t \rightarrow-\infty$.

Proof. From the proof of Lemma 2 it is clear that a closed nonempty subset $\Gamma_{+}$of $\Gamma_{1}$ exists such that if $\left(x_{1}(0), y_{1}(0)\right) \in \Gamma_{+}$, then $\left(x_{1}(t), y_{1}(t)\right) \in R$ for $t>0$. Similarly, a subset $\Gamma_{-}$of $\Gamma_{a}$ exists such that 
if $\left(x_{1}(0), y_{1}(0)\right) \in \Gamma$, then $\left(x_{1}(t), y_{1}(t)\right) \in R$ for $t<0$; we omit the details.

We first prove (a). As in the proof of Lemma 4, it follows that $\xi \eta$ is a nondecreasing function of $t$ for $t>0$, and, in fact, $\xi \eta \rightarrow 0$ as $t \rightarrow+\infty$. (In this paragraph all limits are as $t \rightarrow+\infty$.) Hence $\xi \eta \leqq 0$ for $t>0$. But since $(\xi, \eta)$ satisfies $(4)$, we have, since $\phi \geqq 0, \xi \xi=\xi \eta$ $-\phi \xi^{2} \leqq 0$. Hence $\xi^{2}$ is a nonincreasing function of $t$, and thus $\xi^{2} \rightarrow \lambda_{1}^{2}$ where $\lambda_{1} \geqq 0$. Suppose $\lambda_{1}>0$; then $|\xi| \rightarrow \lambda_{1}$, and in fact $|\xi| \geqq \lambda_{1}$ for $t>0$. From the properties of $h(x, \xi)$, it follows that there exists a constant $\epsilon>0$ such that $h(x(t), \xi(t)) \geqq \epsilon$ for $t>0$. Hence from (4), $|\dot{\eta}|$ $\geqq \epsilon \lambda_{1}$, and we conclude that $|\eta|$ cannot be bounded for $t>0$ as it must be by hypothesis. Hence $\lambda_{1}=0$; i.e., $\xi \rightarrow 0$. From (4) we also have $\eta \dot{\eta}=h \xi \eta \leqq 0$; hence $\eta^{2}$ is nonincreasing and therefore $|\eta| \rightarrow \lambda_{2} \geqq 0$. If $\lambda_{2}>0$, then from (4) and the fact that $\xi \rightarrow 0$, we have $|\xi| \rightarrow \lambda_{2}$. This again leads to a contradiction since $|\xi|$ is bounded for $t>0$. Hence $\lambda_{2}=0$, and finally $(\xi, \eta) \rightarrow(0,0)$.

To prove (b) we first make a change of variable $\tau=-t$ in (4); hence (4) becomes

$$
\begin{aligned}
& \dot{\xi}=-\eta+\phi \xi, \\
& \dot{\eta}=-h \xi
\end{aligned}
$$

where $\xi=\xi(-\tau), \eta=\eta(-\tau)$, and the dot now indicates differentiation with respect to $\tau$. Then in what follows in this paragraph, all limits are as $\tau \rightarrow+\infty$. Since $(\xi \eta)=-Q(\xi, \eta)$ where $Q$ is as in Lemma 4 , it follows that $\xi \eta$ is nonincreasing in $\tau$, and that, as in the previous case, $\xi \eta \rightarrow 0$. Hence $\xi \eta \geqq 0$ for $\tau>0$. From $\left(4^{\prime}\right), \eta \dot{\eta}=-h \xi \eta \leqq 0$; hence $\eta^{2}$ is nonincreasing, and $|\eta| \rightarrow \mu \geqq 0$. If $\mu>0$, then $\xi \rightarrow 0$, and from ( $\left.4^{\prime}\right)$, $|\dot{\xi}| \rightarrow \mu$ which again contradicts the boundedness of $|\xi|$ for $\tau>0$. Hence $\mu=0$; i.e., $\eta \rightarrow 0$. Suppose now $\xi \rightarrow 0$. Then there exist $\delta>0$ and $\tau_{0}>0$ such that $\left|\xi_{0}\right|=\delta$, and if $\delta_{1}$ is such that $|\phi \xi| \geqq 2 \delta_{1}>0$ for $|\xi| \geqq \delta$, then $|\eta|<\delta_{1}$; here $\xi_{0}=\xi\left(-\tau_{0}\right)$. Suppose $\xi_{0}=\delta$; then from $\left(4^{\prime}\right)$, $\xi=-\eta+\phi \xi>\delta_{1}$ when $\tau=\tau_{0}$. We now show that $\xi>\xi_{0}$ for $\tau>\tau_{0}$. If not, then $\xi=\xi_{0}$ for $\tau=\tau_{2}>\tau_{0}$ while $\xi>\xi_{0}$ for $\tau_{0}<\tau<\tau_{2}$. Hence, there exists a $\tau_{1}, \tau_{0}<\tau_{1}<\tau_{2}$, such that for $\tau=\tau_{1}, \xi=0$. Thus by $\left(4^{\prime}\right),-\eta+\phi \xi=0$ for $\tau=\tau_{1}$. But $-\eta+\phi \xi>\delta_{1}$ for $\tau_{0}<\tau<\tau_{2}$ and we have a contradiction. This proves that $\xi>\delta$ for $\tau>\tau_{0}$. Hence, using $\left(4^{\prime}\right)$, we have $\xi>\delta_{1}$ for $\tau>\tau_{0}$ and thus again a contradiction. Hence if $\xi_{0}=\delta$, then $\xi \rightarrow 0$. The argument for the case $\xi_{0}=-\delta$ proceeds similarly; we omit the details. Hence in any case $(\xi, \eta) \rightarrow(0,0)$ as $\tau \rightarrow \infty$ and the proof of the lemma is complete.

Proof of Theorem. From Lemmas 3 and 4 and a general result due to Amerio [3] which states that the unique solution $(x(t), y(t))$ 
of (3) such that $(x(t), y(t)) \in R$ for all $t$ is, in fact, a.p., we conclude the existence of a unique a.p. solution of (3). From Lemma 5, it follows that we may take as $\Sigma_{1}$ the set of solutions $(x(t), y(t))$ of (3) such that $(x(0), y(0)) \in \Gamma_{+}$and as $\Sigma_{2}$ the set of $(x(t), y(t))$ such that $(x(0), y(0)) \in \Gamma_{-}$. Clearly (2) is a system of the form of (3). Hence the theorem follows.

We observe that there exist closed subsets of $\Gamma_{b}$ and $\Gamma_{2}=\Gamma$ $-\left(\Gamma_{1} \cup \Gamma_{a} \cup \Gamma_{b}\right)$ having the same properties as the sets $\Gamma_{-}$and $\Gamma_{+}$ respectively of Lemma 5 .

3. Extension of the theorem. The part of condition (iii) requiring $g(x)>k$ for $x \geqq b$ and $g(x)<-k$ for $x \leqq a$ can be weakened. For example, suppose there exists a region $S$ of the $(x, y)$ plane such that $R \cap S$ is empty, and such that the graph of $y=F(x)$ for $x_{0}<x<x_{1}$ is contained in $S$; here $x_{0}=-\infty$ or $x_{1}=+\infty$ is allowed. Suppose further that for any solution $(x(t), y(t))$ of (2) if $\left(x\left(t_{0}\right), y\left(t_{0}\right)\right) \in S$, then $(x(t), y(t)) \in S$ for $t>t_{0}$. Then the theorem will follow even if possibly $|g(x)| \leqq k$ for $x_{0}<x<x_{1}$. For again, any trajectory of (3) leaving $R$ at some $t=t_{0}$ will not return to $R$ for any $t>t_{0}$, and Lemma 1 and all subsequent lemmas would again follow.

\section{REFERENCES}

1. C. E. Langenhop, $A$ note on almost periodic solutions of nonlinear differential equations, J. Math. Phys. vol. 30 (1959) pp. 126-129.

2. C. E. Langenhop and G. Seifert, Almost periodic solutions of second order nonlinear differential equations with almost periodic forcing, Proc. Amer. Math. Soc. vol. 10 (1959) pp. 425-432.

3. L. Amerio, Soluzioni quasi-periodiche, o limitate, di sistemi differenziali non linearei quasi-periodici o limitati, Ann. Mat. Pura Appl. vol. 39 (1955) pp. 97-119.

Iowa State University of Science and Technology 